\title{
Mobilidade Internacional com Contrato Local: Impactos nas Estratégias e Ações de Recursos Humanos Internacional
}

\author{
International Mobility with Local Contract: Impacts on International Human Resources \\ Strategies and Actions
}

\begin{abstract}
Daniela Farah de Lima ${ }^{1}$ Carlos Roberto Domingues ${ }^{2}$

\section{Resumo}

Para atuação global, as empresas demandam flexibilidade de seus profissionais para mobilidade internacional, entendida como a atuação do trabalhador em outros países. O objetivo desta pesquisa foi analisar os impactos da mobilidade internacional com contrato local nas estratégias e ações de Recursos Humanos $(\mathrm{RH})$ de empresas multinacionais. A abordagem da pesquisa é qualitativa, do tipo descritiva, com uso de estudo de casos múltiplos. Os dados foram obtidos por meio de entrevista semiestruturada com respondentes-chaves da área de $\mathrm{RH}$ de quatro empresas. Os resultados apontaram que são necessárias mais políticas e ações para atender à modalidade de contrato local para: definição das vagas; recrutamento/seleção do profissional global; capacitação e desenvolvimento de competências interculturais e ações de incentivo à carreira internacional. A mobilidade internacional com contrato local tem sido cada vez mais utilizada, principalmente por apresentar menor custo e menor risco para as empresas. A pesquisa também mostrou algumas consequências para os profissionais dessa modalidade comparando-a com a expatriação "tradicional": ela estabelece menor ganho econômico/financeiro; aumenta as incertezas na carreira; transfere maior responsabilidade pela adaptação e inserção (definitiva) na nova cultura, e o processo de avaliação de desempenho exerce maior pressão, dada a impossibilidade de repatriação em caso de insucesso.
\end{abstract}

Palavras-chave: mobilidade internacional; expatriação; gestão internacional de recursos humanos; contrato local de trabalho; carreira internacional.

\begin{abstract}
For global operations, companies demand flexibility from their professionals for international mobility, understood as the workers' performance in other countries. This research aimed to analyze the impacts of international mobility with a local contract on the multinational companies' Human Resources (HR) strategies and actions. The research approach is qualitative, descriptive, using multiple case studies. Data were obtained through semi-structured interviews with HR key-respondents from four companies. The results showed that more policies and actions are needed to meet the local contract modality to define vacancies, recruitment/ selection of the global professional, training and development of intercultural skills, and events encouraging international careers. International mobility with local contracts has been increasingly used, mainly because it presents lower cost and lower risk for companies. The research also showed some consequences for the professionals of this modality when comparing it with the traditional expatriation: it establishes less economic/financial gain; increases career uncertainties; transfers greater responsibility for adaptation and (definitive) insertion in the new culture, and the performance evaluation process puts more pressure, given the impossibility of repatriation in the event of failure.
\end{abstract}

Keywords: international mobility; expatriation; international human resource management; workplace contract; international career. 


\section{INTRODUÇÃO}

De acordo com Biemann e Andresen (2010), quanto mais as empresas expandem sua atuação para outros países, maior se torna a urgência em desenvolver profissionais com competências globais, capacidade de interação eficaz com pessoas de culturas diversas, aptidão para lidar com ambientes competitivos e políticos, e com capacidade para converter instabilidades e incertezas em oportunidades. Para isso, a experiência internacional - por meio da mobilidade internacional - é um instrumento eficaz para o desenvolvimento de tais competências, além desse desenvolvimento ser considerado parte do processo de progressão na carreira. A expatriação (expatriation) é o tipo mais comum de mobilidade internacional e consiste no processo no qual os profissionais trabalham em uma unidade da empresa em um país diferente do seu, tendo sido enviados ao exterior por opção e custeio da empresa, retornando depois de um período para o seu país de origem (Baruch, Dickmann, Altman \& Bournois, 2013).

Outro tipo relevante de mobilidade internacional é a mobilidade de vínculo, denominada por Andresen et al. (2014) de expatriação autoiniciada (self initiated expatriation - SIE), caso do indivíduo que se muda por iniciativa própria para outro país, ou seja, tem uma experiência de carreira internacional independentemente de empregador ou qualquer assistência por parte de alguma organização, por tempo indeterminado. É caracterizado por escolher livremente seu local de destino e o tempo de permanência no lugar (Andresen, Bergdolt, Margenfeld \& Dickmann, 2014; Ariss \& Crowley-Henry, 2013; Biemann \& Andresen, 2010; Cao, Hirschi \& Deller, 2013; Cerdin \& Selmer, 2014). Importante destacar que, apesar da expatriação "tradicional" ser bastante pesquisada, difundida e praticada pelas empresas, o número de expatriados autoiniciados no segundo milênio, segundo Suutari, Brewster, Mäkelä, Dickmann, \& Tornikoski (2018), é maior do que a quantidade de expatriados "tradicionais".

Diante dessa realidade, alinhar as estratégias de gestão de recursos humanos e as estratégias organizacionais é condição essencial a fim de que a empresa alcance os resultados desejados. Para que seja possível a adaptação das organizações a esse ambiente, há consenso entre autores, como Brewster et al. (2010), Lacombe e Albuquerque (2008) e Wood Jr et al. (2011), quanto à atuação da área de RH na definição de estratégias, bem como na formação da equipe de trabalho como fonte de vantagem competitiva. Para Calderón, Guedes e Carvalho (2016), a área se desenvolveu e ganhou destaque com a finalidade de suprir as necessidades de mobilidade internacional e os desafios que essas mudanças proporcionam. Bueno e Freitas (2015) colocam que é de responsabilidade da área de RH fazer com que o processo de mobilidade internacional seja claro, por meio da recepção dos profissionais no local de destino e sua orientação no trabalho, o que ajuda positivamente tanto o empregado quanto o empregador, levando mais rapidamente ao alcance dos resultados almejados.

Como o processo de expatriação "tradicional" é caro e arriscado para as empresas, já que são inúmeros os fatores que concorrem para a volta antecipada ou desistência da missão internacional (Freitas, 2009), elas têm optado por abrir vagas internacionais, em que seus empregados possam concorrer e, se aceitos, encerram o contrato de trabalho no país de origem e iniciam novo vínculo com contrato local no país de destino, que são os denominados expatriados autoiniciados com contrato local (self expatriates with local contract). Outro aspecto relevante a ser considerado em relação ao levantamento bibliográfico previamente realizado relaciona-se à grande quantidade de publicações com foco no trabalhador, e não propriamente na descrição das políticas e das práticas adotadas pelas empresas.

Assim, o objetivo deste artigo é analisar os impactos nas estratégias e ações da área de RH com a intensificação do uso dos contratos locais para mobilidade internacional. Logo, a relevância desta pesquisa é justificada por abordar uma realidade em ascensão no ambiente organizacional, já que, segundo Cao et al. (2013), devido à falta de pessoas qualificadas em muitos países e à crescente demanda por profissionais com habilidades e flexibilidade interculturais, os expatriados autoiniciados são estrategicamente significativos para muitas organizações. Ademais, entende-se que, com este trabalho, é possível contribuir com o preenchimento da lacuna teórica e empírica e estimular novas pesquisas, devido ao escasso material produzido sobre autoexpatriado com contrato local. De acordo com McKenna e Richardson (2007) e Suutari e Brewster (2000), a expatriação autoiniciada é um fenômeno mais recorrente do que a literatura indica.

\section{FUNDAMENTAÇÃO TEÓRICA}

\subsection{Gestão Internacional de Recursos Humanos}

Bueno (2010) relata que a evolução dos processos de tecnologia da informação e o maior acesso e o barateamento do custo dos meios de transporte de longa distância proporcionaram às organizações a expansão de sua atuação, tanto nacional como internacional, por meio do deslocamento de suas operações e de seus empregados. Diante desse cenário, Domingues (2011) afirma que, para tal, as organizações precisam de suporte e direcionamento para suas políticas e práticas, bem como de pessoas adequadamente preparadas e com visão de mundo global.

Na conjuntura internacional, segundo Caligiuri e Colakoglu (2007), a área de RH tem como função desenvolver práticas para o gerenciamento de talentos dos profissionais que irão atuar fora do país de origem, ou seja, gerir carreiras internacionais daqueles que serão responsáveis por manter e difundir o plano estratégico global da empresa. Para Freitas (2005, p. 59), não se deve admitir que “(...) a área de RH de uma empresa globalizada continue a praticar uma 
gestão miúda e arcaica em que o trabalho no exterior é considerado em si como um prêmio e desconsiderar todas as implicações de uma vida profissional expatriada". Assim, a Gestão Internacional de Recursos Humanos (GIRH), segundo Brewster, Sparrow e Vernon (2010), coordena a forma como as organizações internacionais gerenciam seus recursos humanos nos diferentes contextos, sendo seu papel fornecer e permitir soluções globais, regionais e locais de valor agregado e econômicas em uma série de processos centrais de administração de recursos humanos; identificar questões nacionais e internacionais orientadas para o cliente, assim como atender às suas demandas; demonstrar que a conectividade global agrega valor, conhecimento e experiência; e apoiar a estratégia corporativa de gestão de pessoas. Aárea deve gerenciar as demandas de modo que possa assegurar que a organização tenha uma coerência internacional e econômica na forma de gerenciar seus empregados, independentemente de onde estejam alocados, ao mesmo tempo em que deve garantir a compreensão em relação às diferenças e particularidades regionais.

Freitas (2006, p. 50) também cita questões estratégicas, como gerenciamento da diversidade, criação de equipes globais e desenvolvimento da consciência global, e que "quando os responsáveis pela área têm experiência internacional, são mais sensíveis às situações com as quais os profissionais expatriados irão se defrontar e poderão acolher com mais profissionalismo as suas demandas". De tal forma, uma empresa internacionalizada ou em processo de internacionalização está condicionada aos contextos locais - outras legislações, culturas e modos de trabalho - e, por isso, é necessário possuir flexibilidade para lidar com cada situação. A partir desses fatores internos e externos, a GIRH da organização pode ser, conforme Cerdin (2004), baseada em quatro abordagens: i) abordagem etnocêntrica, na qual posições de gestão ou de níveis mais elevados de senioridade são exercidas por profissionais da matriz em regime de mobilidade internacional, havendo pouca ou nenhuma valorização da cultura local; ii) abordagem policêntrica, em que as posições mais estratégicas das subsidiárias são ocupadas com base no particularismo local, ou seja, por profissionais do país onde está instalada a unidade da organização e não há adoção de processos de mobilidade; iii) abordagem geocêntrica, a qual preza pela gestão de carreira global independentemente da origem do profissional, seja ele da matriz, das subsidiárias ou de qualquer outro lugar no mundo; e iv) a abordagem regiocêntrica, em que a gestão da carreira é caracterizada por uma limitação da mobilidade, restrita a certas regiões em particular. A escolha da abordagem a ser adotada pela organização determina como será a gestão de carreira, especialmente em se tratando de carreiras internacionais, bem como o caminho a ser seguido pelas subsidiárias: se dependentes e multiplicadoras da cultura da matriz ou adaptadas à cultura local (Bueno, 2010).

As práticas mais comuns no processo de globalização da área de $\mathrm{GIRH}$, segundo Brewster et al. (2010), são: i) a gestão de pessoas, por intermédio de um conjunto integrado de iniciativas corporativas que visam melhorar a qualificação, a disponibilidade e a flexibilidade de seus profissionais, especialmente aqueles que apresentam alto potencial e que podem ter um impacto positivo e relevante no desempenho organizacional; ii) a identificação das marcas corporativas e globais, ou seja, como as companhias veem sua imagem externa e sua reputação corporativa e a maneira pela qual seus empregados se identificam com a marca e iii) a construção de sistemas de coordenação global em torno das principais competências estratégicas que diferenciam a empresa e levam à vantagem competitiva, através da inclusão das capacidades ou habilidades organizacionais integradas ao desenvolvimento da carreira e/ ou sistemas de gerenciamento do desempenho, sendo que cada uma dessas práticas tem seus próprios desafios, gerenciados no cenário global e considerados em suas particularidades.

Cerdin e Sharma (2014) afirmam que duas atividades são primordiais na GIRH: identificar e atrair indivíduos interessados no trabalho internacional, e motivar e reter as pessoas com talento executivo internacional. Para Muritiba (2009), a GIRH é diferente da gestão de recursos humanos das empresas "domésticas" por atuar em atividades específicas, principalmente relacionadas às funções de capacitação de profissionais que possam vir a atuar nas subsidiárias, como a administração dos processos de mobilidade internacional, programas de rotatividade de funções ou países (para que os empregados entendam as operações internacionais), a administração de programas de treinamentos internacionais, relações com governos e agências de países estrangeiros, questões tributárias, de realocação e socialização internacionais, entre outras. Logo, os subsistemas de GIRH sofrem mudanças e adaptações diante do contexto internacional, de modo que a seleção deve apresentar maior abrangência, haja vista a complexidade dos cargos e da estrutura da organização, bem como o mercado de trabalho, que não está mais restrito apenas a profissionais locais (Domingues, 2011).

A gestão da remuneração e dos benefícios é uma importante atribuição, já que a política de remuneração é, muitas vezes, um dos incentivos para que o profissional aceite uma atribuição internacional. Também o sistema de avaliação de desempenho deve contemplar o planejamento em longo prazo da organização, por meio de métodos combinados de avaliação no local de atuação do empregado e na matriz, assim como do processo de comunicação, garantindo o maior número de informações e o maior número de pessoas envolvidas, e os programas de treinamento, capazes de desenvolver a capacidade de superação de desafios, culturais, sociais, econômicos e/ou burocráticos (Brewster et al., 2010).

Domingues (2011) afirma que a área, em conjunto com os empregados, deve elaborar e planejar a carreira do profissional global, que a gestão de carreira e os programas de gestão de recursos humanos (internacional ou doméstica) são elementos primordiais, tanto para os indivíduos quanto para as organizações, e que se deve pensar na carreira internacional como um todo, de maneira a possibilitar e expandir os processos de mobilidade internacional. 


\subsection{Tipos de Mobilidade Internacional}

Collings, Scullion e Morley (2007) apontaram, há mais de uma década, que estão surgindo outros modelos de atribuições internacionais, as "formas alternativas" ou "atribuições internacionais não padrão". Essas mudanças decorrem, principalmente, da necessidade de contenção de despesas e de restrições em relação à seleção de expatriados que, muitas vezes, não aceitam a atribuição fora do país em virtude de questões familiares, como a carreira do/a cônjuge, parentes idosos ou dependentes, filhos etc. Variados termos são utilizados para distinguir essas modalidades, o que, segundo Baruch et al. (2013), é necessário a fim de se conhecer as peculiaridades do trabalho em países estrangeiros e como isso influencia as carreiras, auxilia na construção da identidade dessas pessoas, facilita a contratação nas organizações e identifica as expectativas e os contratos psicológicos estabelecidos. No Quadro 1 são resumidos os tipos de mobilidade internacional e suas diferenças, segundo a finalidade, a natureza e a duração da atribuição.

Quadro 1 - Tipos de Mobilidade Internacional

\begin{tabular}{|c|c|c|c|}
\hline TIPO & FINALIDADE & NATUREZA / DURAÇÃO & AUTORES \\
\hline EXPATRIAÇÃO & $\begin{array}{l}\text { Efetivar, nas subsidiárias, as diretrizes } \\
\text { da matriz; desenvolver liderança de } \\
\text { executivos; expandir e aumentar a } \\
\text { atuação nos mercados internacionais; } \\
\text { Internacionalizar a gestão. }\end{array}$ & $\begin{array}{l}\text { - Iniciada pela organização. } \\
\text { - } 06 \text { meses a } 05 \text { anos }\end{array}$ & $\begin{array}{l}\text { Bueno (2010) } \\
\text { Gallon; Antunes (2016) } \\
\text { Gallon; Bitencourt (2016) } \\
\text { Gallon; Scheffer; Bitencourt (2013) } \\
\text { Inkson, Arthur, Pringle, Barry (1997) }\end{array}$ \\
\hline FLEXPATRIAÇÃO & $\begin{array}{l}\text { Executar uma atribuição específica, } \\
\text { em curto prazo, que requer alta } \\
\text { especialização. }\end{array}$ & $\begin{array}{l}\text { - Iniciada pela organização } \\
\text {-Não determinada, depende } \\
\text { de casos pontuais. }\end{array}$ & $\begin{array}{l}\text { Collings; Scullion; Morley (2007) } \\
\text { Mayerhofer; Hartmann; Herbert } \\
(2004) \\
\text { Welch; Welch; Worm (2007) }\end{array}$ \\
\hline $\begin{array}{c}\text { MOVIMENTO } \\
\text { TRANSFRONTEIRIÇO }\end{array}$ & $\begin{array}{l}\text { Suprir a necessidade de mão de } \\
\text { obra qualificada nos grandes centros } \\
\text { urbanos. }\end{array}$ & $\begin{array}{l}\text { - Iniciado pelo indivíduo } \\
\text { - Diária ou semanal. }\end{array}$ & $\begin{array}{l}\text { Buch; Schmidt; Niebuhr (2009) } \\
\text { Decoville et al. (2013) }\end{array}$ \\
\hline $\begin{array}{c}\text { ATRIBUIÇÃO DE CURTO } \\
\text { PRAZO }\end{array}$ & $\begin{array}{l}\text { Aprimorar e desenvolver carreiras; } \\
\text { transferir tecnologia e conhecimento; } \\
\text { implantar projetos, solucionar problemas } \\
\text { pontuais e gerenciar uma operação em } \\
\text { particular; criar e manter relacionamento } \\
\text { com clientes, fornecedores e } \\
\text { profissionais locais. }\end{array}$ & $\begin{array}{l}\text { - Iniciado pela organização } \\
\text { - } 01 \text { a } 12 \text { meses }\end{array}$ & $\begin{array}{l}\text { Salleh; Koh (2013) } \\
\text { Tahvanainen; Welch; Worm (2005) }\end{array}$ \\
\hline IMPATRIAÇÃO & $\begin{array}{l}\text { Desenvolver gestores por meio da } \\
\text { transferência de profissionais das } \\
\text { subsidiárias para a matriz; estabelecer } \\
\text { e/ou fortalecer o vínculo entre a matriz e } \\
\text { as subsidiárias; transferir conhecimento. }\end{array}$ & $\begin{array}{l}\text { - Iniciada pela organização; } \\
\text { - Não foi mencionado } \\
\text { período específico, porém } \\
\text { trata-se de uma atribuição } \\
\text { com duração determinada. }\end{array}$ & $\begin{array}{l}\text { Harvey; Kiessling; Moeller (2011) } \\
\text { Harvey; Speier; Novicevic (1999) } \\
\text { Maley; Moeller; Harvey (2015) }\end{array}$ \\
\hline MIGRAÇÃO & $\begin{array}{l}\text { Buscar melhores condições de emprego, } \\
\text { segurança e, em alguns casos, até } \\
\text { sobrevivência. }\end{array}$ & $\begin{array}{l}\text { - Iniciada pelo indivíduo. } \\
\text { - Indefinida, geralmente de } \\
\text { caráter permanente. }\end{array}$ & $\begin{array}{l}\text { Haas (2005) } \\
\text { Zikic (2015) }\end{array}$ \\
\hline $\begin{array}{l}\text { EXPATRIAÇÃO AUTO } \\
\text { INICIADA }\end{array}$ & $\begin{array}{l}\text { Aprimorar habilidades profissionais e } \\
\text { pessoais; criar redes de contatos; } \\
\text { formar verdadeiros profissionais globais. }\end{array}$ & $\begin{array}{l}\text {-Iniciada pelo indivíduo. } \\
\text {-Indefinida. } \\
\text { - Com contrato local }\end{array}$ & $\begin{array}{l}\text { Andresen et al. (2014) } \\
\text { Ariss; Crowley-Henry (2013) } \\
\text { Biemann; Andresen (2010) }\end{array}$ \\
\hline
\end{tabular}

Fonte: Os autores com base nas fontes supracitadas e usando a classificação de Tahvanainen, Welch e Worm (2005).

Devido à escassa literatura sobre a expatriação autoiniciada com contrato local, para sustentação desta pesquisa, será detalhada a expatriação autoiniciada em contraponto com a expatriação "tradicional". Segundo Cerdin e Selmer (2014), a self-initiated expatriation (SIE) está emergindo rapidamente, porém, ao se realizar um levantamento bibliográfico sobre o tema, verificou-se que ainda não há um consenso ou clareza em um único constructo que dê conta de todos os aspectos que envolvem esse tipo de mobilidade internacional, diferentemente da literatura sobre a expatriação "tradicional".

A característica mais imediata que difere expatriados "tradicionais" dos self-initiated expatriates é quem é o responsável pela iniciativa de trabalhar internacionalmente. Para Inkson et al. (1997) e Cerdin e Selmer (2014), a expatriação autoiniciada resulta de uma decisão unicamente do indivíduo de se deslocar para outro país, com a expectativa de obter vantagens individuais e/ou familiares e autorrealização. 
Doherty, Richardson e Thorn (2013) contradizem a afirmação quanto à permanência por tempo indeterminado ser uma característica da expatriação autoiniciada. Para esses autores, o limite temporal é fundamental para a compreensão do SIE, uma vez que a delimitação de tempo de permanência é importante: enquanto o indivíduo permanecer temporariamente fora de seu país de origem, ele pode ser considerado como expatriado autoiniciado, mas, em caso de mudança permanente, ele passa a ser um migrante. Assim, duas características essenciais descrevem os profissionais autoexpatriados: a recolocação profissional em outro país e a iniciativa própria da mobilidade internacional (Doherty et al., 2013).

Em um trabalho de Cerdin e Selmer (2014), quatro critérios são usados para a descrição do expatriado autoiniciado: i) a própria iniciativa para a mudança para outro país de sua escolha; ii) a intenção (ou já obtenção) de emprego regular e formal no local de destino; iii) a intenção de permanecer apenas temporariamente (pois, ao contrário dos migrantes, o SIE pretende retornar ao país de origem) e iv) habilidades e qualificação profissionais. De acordo com os autores, somente quando os quatro critérios ocorrem simultaneamente tem-se representado o expatriado autoiniciado. Os SIE não se beneficiam, a priori, de qualquer auxílio financeiro para o deslocamento, habitação ou outras formas de assistência que poderiam facilitar a integração no país anfitrião, o que pode representar um desafio particular para esses profissionais que não têm, como regra, o custeio de suas despesas patrocinado pelas organizações. Ao mesmo tempo, esses indivíduos demonstram maior habilidade em adaptar-se ao país estrangeiro, interagir com a comunidade e aprender a língua local, quando comparados aos expatriados "tradicionais". E, conforme destacado por Andresen et al. (2014), ao contrário do expatriado "tradicional", a decisão de contratar um SIE é sempre de um novo empregador, ou seja, será celebrado um novo contrato de trabalho no exterior, que denominará o expatriado como autoiniciado com contrato local.

Alguns aspectos são listados na literatura como motivadores para a expatriação autoiniciada: os SIE apresentam alta iniciativa e motivação pessoal em relação à internacionalização, além disso, situações relacionadas ao mercado de trabalho e possibilidades de carreira também são aspectos relevantes em relação à decisão dessas pessoas ao saírem de seu país de origem, assim como mudanças no estilo de vida, oportunidades de aprendizado, desenvolvimento pessoal e profissional, questões familiares e/ou financeiras, busca por novos desafios, oportunidades culturais e de viagem, ambiente político, expectativas em relação à realização de trabalhos que lhe despertem interesse, desenvolvimento pessoal proporcionado pela experiência "autodirigida", busca por aventura, oportunidades para desenvolvimento de familiares (por exemplo, educação dos filhos), melhor qualidade de vida e criação de redes de contatos (Ariss \& Crowley-Henry, 2013; Biemann \& Andresen, 2010; Inkson et al., 1997; Mckenna \& Richardson, 2007; Thorn, 2009).

Os expatriados autoiniciados representam ganhos significativos para as organizações em decorrência de sua boa compreensão de mercado, tanto local quanto internacional, língua e culturas, com custo bastante inferior ao expatriado "tradicional", considerando gastos com viagem, salário, tributação, entre demais questões financeiras e tributárias (Ariss \& Crowley-Henry, 2013). A experiência de trabalho internacional por meio dos self-initiated expatriates, segundo Biemann e Andresen (2010), representa uma alternativa à carreira no contexto global, visto que é autogerenciada e esses indivíduos podem ser considerados como verdadeiros profissionais globais, com disposição e disponibilidade de se mudar para vários lugares ao longo do tempo.

Em um estudo realizado por Chen e Shaffer (2017), os expatriados autoiniciados que estavam mais motivados em relação ao trabalho no exterior estavam mais propensos a demonstrar níveis elevados de comprometimento organizacional e com a comunidade, e também compromisso afetivo, sendo a situação pessoal e as características do local de trabalho forças de atração para que esses profissionais se vinculem efetivamente às suas organizações. Por outro lado, Hussain e Deery (2018) argumentam que a disposição e a tendência de estar sempre em movimento observada nos SIE pode tornar a retenção dos empregados mais desafiadora para as empresas.

Os expatriados autoiniciados, de acordo com Cao et al. (2013), representam indivíduos conscientes acerca das oportunidades e progressão de suas carreiras, por isso buscam seus objetivos em outros países. Assim, segundo os autores, esses profissionais se mostram flexíveis e não dependentes da organização para seu desenvolvimento, aspecto reforçado por Suutari et al. (2018), que afirmam que os expatriados autoiniciados são agentes do próprio desenvolvimento profissional, ao contrário dos expatriados "tradicionais", fortemente dependentes das estruturas de carreira nas organizações. Collings et al. (2007) acrescentam que a principal consequência do aumento do número de expatriados autoiniciados refere-se ao fato de que as organizações lançam mão desses profissionais para preencherem posições estratégicas em suas subsidiárias, com menor custo em comparação aos expatriados "tradicionais", usando aqui o conceito de expatriado autoiniciado com contrato local. É interessante pontuar que a maioria dos trabalhos aponta vantagens para as empresas com a contratação do autoexpatriado e pouco se comenta sobre os ganhos e perdas para os indivíduos em comparação com as modalidades mais tradicionais da mobilidade internacional.

Uma diferenciação dos expatriados autoiniciados e daqueles intraorganizacionais (Intra-SIE), que continuam trabalhando na mesma organização, porém com um novo contrato de trabalho no exterior, com contrato local, objeto de estudo dessa pesquisa, é apresentada por Andresen et al. (2014), que consideram o expatriado autoiniciado com contrato local aquele profissional que, voluntariamente e por decisão pessoal, encerra seu vínculo empregatício no seu país de origem e inicia um novo contrato em uma unidade da mesma organização na qual trabalha.

A complementariedade entre os estudos de Collings et al. (2007) e de Anddresen et al. (2014) representa um marco na elaboração do constructo e clarificam os elementos que permitem que se aprofundem os estudos em 
relação ao gerenciamento da carreira. Partindo-se dessa distinção entre os empregados internacionais, há também a possibilidade do desenvolvimento de políticas e práticas de $\mathrm{RH}$ particulares e específicas para cada grupo.

\section{PROCEDIMENTOS METODOLÓGICOS}

A abordagem desta pesquisa é qualitativa e, no que diz respeito aos objetivos, trata-se de uma pesquisa descritiva. Foi realizado um estudo de casos múltiplos, com quatro empresas de diferentes setores. As empresas pesquisadas foram selecionadas de acordo com o critério de praticarem a mobilidade internacional do tipo expatriação autoiniciada com contrato local.

Entre as organizações respondentes, foi inserida uma organização cujaentrevista foi realizada no RH global, na matriz, sendo essa caracterizada como Empresa A. Tal situação se dá para ilustrar de forma mais contundente as situações entre o RH global e o RH nas subsidiárias, não havendo com isso nenhuma intenção de comparação entre matriz e subsidiária, mas sim apresentar as realidades encontradas. No Quadro 2 são apresentadas as características das empresas:

Quadro 2 - Empresas Pesquisadas

\begin{tabular}{|c|c|c|c|c|}
\hline & $\begin{array}{c}\text { EMPRESA A } \\
\text { (matriz) }\end{array}$ & $\begin{array}{c}\text { EMPRESA B } \\
\text { (subsidiária) }\end{array}$ & $\begin{array}{c}\text { EMPRESA C } \\
\text { (subsidiária) }\end{array}$ & $\begin{array}{c}\text { EMPRESA D } \\
\text { (subsidiária) }\end{array}$ \\
\hline Ramo & Alimentício & Eletrodomésticos & Construção Civil & Automotivo \\
\hline Origem & Latinoamericana & Europeia & Europeia & Europeia \\
\hline Atuação & 50 países & 150 países & 12 países & 90 países \\
\hline Número de empregados & 105.000 & 55.000 & 88.500 & 40.000 \\
\hline
\end{tabular}

Fonte: Dados da pesquisa.

Em relação aos entrevistados, o critério de escolha foi baseado no conceito de respondente-chave, ou seja, foram identificados os profissionais ligados à área de $\mathrm{RH}$ das empresas pesquisadas que respondiam pela definição e/ou implementação de políticas e ações da área para a mobilidade internacional. Sendo assim, foram entrevistados quatro gestores e analistas de $\mathrm{RH}$, que são responsáveis por conduzir, acompanhar e/ou avaliar o processo de mobilidade internacional. No Quadro 3 são sintetizadas as características dos entrevistados:

Quadro 3 - Caracterização dos Entrevistados

\begin{tabular}{|l|l|l|l|l|}
\hline & \multicolumn{1}{|c|}{ EMPRESA A } & \multicolumn{1}{c|}{ EMPRESA B } & \multicolumn{1}{c|}{ EMPRESA C } & \multicolumn{1}{c|}{ EMPRESA D } \\
\hline Cargo & Coordenador de RH & Analista de RH & Gerente de RH & Analista de RH \\
\hline Formação & Psicologia e Direito & Administração & Psicologia & Educação Física \\
\hline Atuação na profissão & Desde 2010 & Desde 2011 & Desde 1998 & Desde 1985 \\
\hline Atuação na organização & Desde 2014 & Desde 2015 & Desde 2011 & Desde 1998 \\
\hline Posição hierárquica & $\begin{array}{l}\text { Responde ao Diretor } \\
\text { de RH }\end{array}$ & $\begin{array}{l}\text { Responde ao } \\
\text { Gerente de RH }\end{array}$ & $\begin{array}{l}\text { Responde ao Diretor } \\
\text { de RH }\end{array}$ & $\begin{array}{l}\text { Responde ao Diretor } \\
\text { de RH }\end{array}$ \\
\hline $\begin{array}{l}\text { Ramo de atuação da } \\
\text { organização }\end{array}$ & Alimentício & $\begin{array}{l}\text { Indústria de } \\
\text { Eletrodomésticos }\end{array}$ & $\begin{array}{l}\text { Varejista em } \\
\text { construção civil }\end{array}$ & Automobilístico \\
\hline
\end{tabular}

Fonte: Dados da pesquisa.

Foi elaborado um roteiro de perguntas para as entrevistas, detalhado no Apêndice I. As entrevistas tiveram uma duração média de 1 hora e foram, posteriormente, transcritas com o consentimento dos entrevistados. A análise das informações foi feita usando-se a técnica de análise de conteúdo, seguindo os preceitos de Bardin (2011), e partindo de categorias de análise identificadas a priori, de acordo com o referencial teórico, e que embasaram o roteiro de entrevista. As categorias temáticas de análise são: i) tipos de mobilidade internacional, ii) RH para atuação doméstica e internacional, iii) cargos eletivos para a mobilidade internacional, iv) seleção de profissionais para a mobilidade internacional, v) controle da área de $\mathrm{RH}$ em relação ao contrato local, vi) gerenciamento do contrato local, vii) carreira dos expatriados autoiniciados com contrato local, viii) suporte do RH para o contrato local, ix) dificuldades e desafios do contrato local, $\mathrm{x}$ ) desvinculação do $\mathrm{RH}$ e o profissional com contrato local, e xi) expectativas futuras para o contrato local. 


\section{APRESENTAÇÃO E ANÁLISE DOS DADOS}

Foi indagado aos entrevistados se é feita a gestão de RH separada entre empregados do próprio país e os expatriados, pois, conforme Bueno (2010), as empresas, ao atuarem no ambiente global, podem criar um subsistema específico de RH para a mobilidade/gestão internacional. Conforme Brewster et al. (2010), esta deve garantir: coerência na forma de fazer a gestão de seus empregados; compreender as diferenças e particularidades de cada local onde estão as subsidiárias; identificar o que funciona de um lugar para outro; detectar, atrair, motivar e reter indivíduos interessados e com talento para o trabalho internacional. De acordo com as respostas, nas Empresas A, $\mathrm{B}$ e $\mathrm{C}$, a responsabilidade pelos processos de mobilidade internacional cabe à subárea de Remuneração, que cuida também de todas as políticas de salário, de movimentação, de promoção, de carreira e de descrição de cargos. Essas organizações entendem que a mobilidade internacional é uma movimentação profissional, por isso essa área é a responsável pelo processo. Já a Empresa $\mathrm{D}$ trata da expatriação "tradicional" distintamente do contrato local em termos de responsabilidade por cada processo. Existe uma subárea de mobilidade internacional que cuida especificamente de expatriação, e o contrato local cabe ao RH de origem fazer a rescisão do contrato de trabalho e ao RH do país demandante receber esse profissional de acordo com a legislação e a política da organização no país de destino.

\subsection{Cargos Eletivos para a Mobilidade Internacional}

Visto que, como afirmaram Biemann e Andresen (2010), a partir do momento em que as organizações expandem suas operações para outros países há a necessidade de atuação de profissionais com competências consideradas globais e capacidade de lidar com culturas e ambientes diversos, foi indagado sobre quais cargos são eletivos para a mobilidade internacional com contrato local e quais são os objetivos com o processo. Nenhuma das quatro organizações pesquisadas estabelece formalmente quais cargos são eletivos para o contrato local, afirmando que até mesmo cargos técnicos são possíveis de serem ocupados nessa modalidade. Quanto aos objetivos, na Empresa A faz parte da estratégia de expansão dos negócios (novas subsidiárias/aquisições), sendo essa modalidade usada para a implementação da cultura e dos processos da matriz. A Empresa B preza pela diversidade e é guiada pela premissa das "melhores pessoas nos locais mais adequados", assim, o contrato local promove o ambiente multicultural e a troca de conhecimento almejado pela organização. Para a Empresa C, a mobilidade internacional com contrato local possibilita a replicação da cultura e a gestão da matriz em suas subsidiárias e, ao trazer profissionais de outras localidades, favorece a política organizacional de cultura global. Na Empresa D, os objetivos estão relacionados ao desenvolvimento dos empregados, desenvolvimento de um projeto específico e transferência de tecnologia (principalmente).

\subsection{Seleção de Profissionais para a Mobilidade Internacional}

Todas as empresas têm processos de recrutamento e seleção similares entre si no que diz respeito à: divulgação das vagas globais, quem poderá acessar essa informação e a forma de candidatura. Utilizam-se meios eletrônicos para divulgação e suas respectivas possibilidades de controle de acesso e forma de confidencialidade, já que a chefia não tem acesso aos candidatos inscritos e, apesar dos entrevistados relatarem que todos os empregados podem se candidatar e concorrer às vagas internacionais, sabe-se que nem todos têm acesso aos canais de divulgação usados. A candidatura ocorre por meio de mídias eletrônicas nas quais o interessado, ao ter contato com a vaga, pode preencher os formulários necessários para o $\mathrm{RH}$ contratante.

\subsection{Carreira dos Expatriados Auto Iniciados com Contrato Local}

Autores como Brewster et al. (2010), Dickmann et al. (2008), Domingues (2011), Freitas (2009) e Gallon et al. (2013) consideram que a mobilidade internacional, seja qual tipo for, é bastante relevante na gestão de carreira, um caminho para conquista de experiência e desenvolvimento. Assim, questionou-se aos entrevistados acerca da carreira dos expatriados autoiniciados com contrato local. Para todas elas, nesse tipo de mobilidade, a gestão da carreira passa a ser de responsabilidade do país contratante, seguindo as diretrizes gerais da organização, ou seja, a partir do momento da ida do profissional para outro país, a área de RH da origem não tem mais qualquer ação sobre a progressão ou o desenvolvimento de carreira desse trabalhador.

\subsection{Gerenciamento do Contrato Local}

Em termos de gerenciamento dos processos que envolvem os profissionais globais, todas as empresas fazem o acompanhamento do expatriado "tradicional", fato que não acontece em relação ao contrato local e contraria, em parte, a literatura, como Brewster et al. (2010), que dizem que as organizações devem socializar as ações e o comportamento de seus profissionais, através da gestão de $\mathrm{RH}$, cuidado com sua autoimagem e desenvolvimento de diferenciais competitivos. Nas quatro empresas os processos são similares: há um programa único de gestão de carreiras que 
contempla planos de desenvolvimento individual e, após a mudança do expatriado autoiniciado com contrato local, todo o acompanhamento e desenvolvimento fica sob responsabilidade do RH do país hospedeiro. Na maioria dos casos, os profissionais se candidatam para cargos superiores do que exercem no Brasil e no país contratante continuam a participar do plano de desenvolvimento individual, porém sem qualquer relação com o RH de origem.

\subsection{Suporte do RH para o Contrato Local}

Quanto ao suporte do $\mathrm{RH}$ oferecido ao expatriado com contrato local, este difere do que é oferecido ao expatriado "tradicional". Na Empresa A, apesar de não possuir uma política estruturada e formal em relação ao contrato local, é oferecido suporte ao empregado, assemelhando-se em termos financeiros aos benefícios concedidos ao expatriado "tradicional". Apesar de não ser um procedimento oficialmente estabelecido, o RH do país hospedeiro auxilia em todo o processo de transferência, desde auxílio com a documentação para retirada do visto e autorização de trabalho no exterior, pagamento de despesas de viagem, mudança e moradia nos primeiros anos de atuação. Na Empresa B, na expatriação "tradicional", o RH participa de todo o processo, desde o convite até a preparação da documentação até a ida do profissional, inclusive através de apoio aos familiares, e paga, como diferencial, um valor financeiro denominado de "variação cambial" como forma de garantir o poder de compra do expatriado durante sua atribuição internacional. No contrato local, os benefícios dependem de cada projeto, mas geralmente a empresa arca com as despesas de viagem e mudança para o profissional e sua família, curso de idiomas, vindas para o Brasil durante os dois primeiros anos, e há casos de pagamento de aluguel por tempo determinado.

Na Empresa C, o expatriado autoiniciado com contrato local, ao ser selecionado para a vaga em outro país, é desligado da empresa como forma de ajuda, para que receba as verbas rescisórias e também recebe ajuda com documentação e visto, por meio de consultoria especializada. Outros benefícios são negociados dependendo de cada caso, já se tratando do expatriado "tradicional", existe uma política de benefícios padronizada, desde o pagamento de aluguel, escola para os filhos e curso de idiomas. Por fim, na Empresa D, o respondente foi bastante enfático ao responder: "completamente diferente". O contrato local recebe os benefícios locais do país para o qual ele está indo; em alguns casos, dependendo da localidade, existe um "plus" oferecido pela organização, como ajuda de custo com mudança, passagem aérea, pagamento de moradia por alguns meses, porém não se trata de uma política formal aplicada a todos os empregados. Comparando os dois tipos de mobilidade internacional praticados, entende-se que a expatriação "tradicional" é um interesse mútuo da organização e do profissional, há um convite por parte da empresa; já o contrato local trata-se de iniciativa do próprio trabalhador.

Esses dados encontrados corroboram Suutari e Brewster (2000) e Hussain e Deery (2018), que dizem haver uma diferenciação relevante entre os expatriados "tradicionais" e os SIE quanto aos benefícios e ganhos financeiros, sendo que, enquanto os primeiros recebem da organização diversos prêmios e bonificações ao assumirem uma atribuição internacional, os segundos ficam sujeitos, na maioria das vezes, exclusivamente às condições locais para onde estão indo, o que pode significar um desafio a mais para esses profissionais, assemelhando-se aos autoexpatriados. Além disso, Brewster et al. (2010) e Gallon e Antunes (2016) descrevem que o processo de expatriação "tradicional" segue diferentes momentos: preparação, ida, ajustamento, atuação em si e a repatriação. Já no caso do expatriado autoiniciado com contrato local, a decisão é considerada como unicamente do profissional, que se muda por conta própria, independentemente de qualquer assistência por parte da organização (Andresen et al., 2014; Ariss \& CrowleyHenry, 2013; Biemann \& Andresen, 2010; Cerdin \& Selmer, 2014); Inkson et al., 1997).

\subsection{Desvinculação do RH e o Profissional com Contrato Local}

Com relação à desvinculação da área ao assumir a atribuição internacional com contrato local, houve diferença dos achados empíricos com relação à literatura: por exemplo, Collings et al. (2007) dizem ser função da área de RH garantir que cada tarefa internacional tenha metas claramente definidas e avaliar o desempenho do trabalhador e o retorno do investimento na atribuição internacional. Ademais, diferentes tipos de mobilidade acarretam em especificidades quanto a impostos, segurança social, vistos e questões legais de entrada e permanência em cada país. No caso de SIE com contrato local, nas quatro empresas pesquisadas não há orientação, acompanhamento ou supervisão por parte da área de $\mathrm{RH}$ no local de onde está partindo o empregado após a mudança; ele irá assimilar alguma possível diferença ou mudança nos processos organizacionais quando assumir sua nova função ou por conversas informais.

\subsection{Dificuldades e Desafios do Contrato Local}

Também se buscou identificar as principais ou as maiores dificuldades ou desafios em relação à expatriação autoiniciada com contrato local e quais são as ações para enfrenta-los. O Quadro 6 resume as principais dificuldades ou desafios relacionados ao contrato local que foram relatados pelos entrevistados: 
Quadro 6 - Principais Dificuldades do Contrato Local

\begin{tabular}{|l|l|l|l|}
\hline \multicolumn{1}{|c|}{ EMPRESA A } & \multicolumn{1}{|c|}{ EMPRESA B } & \multicolumn{1}{c|}{ EMPRESA C } \\
\hline $\begin{array}{l}\text { (1) Remuneração } \\
\text { (2) Adaptação do } \\
\text { profissional e/ou da família }\end{array}$ & $\begin{array}{l}\text { (1) Visto definitivo de } \\
\text { permanência no país } \\
\text { hospedeiro }\end{array}$ & $\begin{array}{l}\text { (1) Dúvidas relativas ao IR no Brasil } \\
\text { (2) Adaptação do profissional e/ou } \\
\text { da família }\end{array}$ & $\begin{array}{l}\text { (1) Visto definitivo de permanência no no } \\
\text { país hedeiro } \\
\text { (2) Dúvidas relativas ao IR no Brasil } \\
\text { (3) Adaptação dos familiares }\end{array}$ \\
\hline
\end{tabular}

Fonte: Dados da pesquisa.

Com exceção da Empresa $B$, nas outras três organizações foram relatados problemas relacionados à adaptação e, apesar disso, não há procedimentos ou ações formais para resolução dessa questão. Para os respondentes, tratase de aspectos pontuais e que as empresas não têm muito que fazer para sanar tais adversidades. Em contrapartida, os ganhos para as organizações são bastante relevantes, já que com as mudanças entre as diferentes unidades os profissionais adquirem cada vez mais experiência e know how sobre o negócio da empresa, diminuindo custos com capacitação, treinamento e desenvolvimento e, ao mesmo tempo, aumentando a eficácia e a eficiência de seu trabalho. Ratifica essa percepção o fato de as pesquisas, tanto as teóricas quanto o resultado deste trabalho, apontarem para o crescimento da mobilidade internacional autoiniciada com contrato local, bem como a tendência de substituição ou até mesmo de extinção da expatriação "tradicional", visto que o contrato local pode trazer os mesmos resultados estratégicos a custos significativamente menores.

\subsection{Expectativas futuras para a Expatriação Auto Iniciada com Contrato Local}

Cerdin e Selmer (2014) afirmam que o número de expatriados autoiniciados deverá aumentar nos próximos anos, juntamente com a contínua globalização do mercado de trabalho. Confirmando a teoria, todos os participantes desta pesquisa responderam afirmativamente quanto ao crescimento e à relevância da expatriação autoiniciada com contrato local. Segundo o respondente da Empresa A, todas as formas de mobilidade internacional são bemvindas e recomendadas para cargos estratégicos, especialmente o contrato local, por possibilitar maior flexibilidade. Para a Empresa B, "o contrato local faz todo o sentido, é um meio sem volta, principalmente por questões de custo". Dado semelhante foi constatado com o profissional entrevistado da Empresa C, que afirmou não ter como voltar nessa tendência, e na Empresa D acredita-se que "os contratos locais vão aumentar, é uma tendência de futuro. Os custos de expatriação são muito altos e a tendência é acabar".

\section{CONCLUSÃO}

Apesar de ainda pouco comentada na literatura, a expatriação autoiniciada com contrato local tem tomado cada vez mais o espaço das modalidades tradicionais de mobilidade internacional nas empresas estudadas, que demonstram a intenção de continuar nesse caminho com o objetivo de diminuição de custos e de preparação de seus profissionais. Apesar dos benefícios já percebidos pelas empresas, é preciso desenvolver/melhorar políticas e ações voltadas para definição e acesso às vagas internacionais; recrutamento/seleção; capacitação e desenvolvimento de competências interculturais; e ações de incentivo e acompanhamento da carreira internacional.

Os achados desta pesquisa sobre o contrato local se aproximam do que já vem sendo discutido sobre as motivações (por parte dos profissionais) e os benefícios percebidos (por parte das empresas) com a expatriação autoiniciada com contrato local. Observa-se, no entanto, que falta analisar em mais profundidade as consequências para os profissionais quando se compara com a expatriação "tradicional": o contrato local estabelece menor ganho econômico/financeiro; aumenta as incertezas na carreira; transfere maior responsabilidade pela adaptação e inserção (que pode ser definitiva) na nova cultura, além do que o processo de avaliação de desempenho tem outra conotação, como fator de pressão, dada a impossibilidade de repatriação em caso de insucesso. Portanto, nessa modalidade, nota-se um aumento da responsabilidade do profissional enquanto os benefícios diminuem.

Por meio desta pesquisa pode-se afirmar que, quanto às políticas e práticas das áreas de Recursos Humanos no que diz respeito à expatriação autoiniciada com contrato local, considerando as quatro empresas pesquisadas, as estratégias de $\mathrm{RH}$ são determinadas pela matriz e praticadas nas diferentes unidades, mesmo que, em alguns casos, haja relativa autonomia e adaptação à cultura local em cada um dos países onde atua, o caso da Empresa C.

Em termos de impactos organizacionais, os ganhos da empresa são bastante relevantes, já que, com as mudanças entre as diferentes unidades, os profissionais adquirem cada vez mais experiência e know how sobre o negócio da empresa, diminuindo custos e impactando nas estratégias de ação da área de RH com programas de recrutamento e seleção, capacitação, treinamento e desenvolvimento e, ao mesmo tempo, aumentando a eficácia e a eficiência de seu trabalho. Ratifica essa percepção o fato de diferentes pesquisas, inclusive esta, apontarem para o crescimento da mobilidade internacional do tipo expatriação autoiniciada com contrato local, bem como a tendência de 
substituição ou até mesmo de extinção da expatriação "tradicional", visto que o contrato local pode trazer os mesmos resultados estratégicos a custos significativamente menores.

Mais do que a questão dos custos menores, destaca-se a transferência quase que total da responsabilidade do sucesso da mobilidade internacional para o profissional, visto que este, ao não ter a garantia do retorno ao trabalho no país de origem, irá se esforçar e se dedicar ainda mais para que o resultado de sua mudança seja positivo e satisfatório, tanto para ele e sua família (se for o caso) quanto para a organização, pois, caso contrário, ele será desligado da empresa. Sendo assim, o empregado, ao assumir a atribuição internacional do tipo contrato local, poderá se adaptar melhor e mais rapidamente ao país para o qual está indo do que na expatriação "tradicional".

Como estudos futuros, sugerem-se pesquisas que analisem a expatriação autoiniciada com contrato local na perspectiva dos profissionais que passaram ou estão passando por essa experiência, e também sobre o quanto o aumento desse tipo de mobilidade internacional impacta e reconfigura as relações no mundo do trabalho nos cenários nacional e global.

\section{Referências}

Andresen, M., Bergdolt, F., Margenfeld, J., \& Dickmann, M. (2014). Addressing international mobility confusion: developing definitions and differentiations for self-initiated and assigned expatriates as well as migrants. The International Journal of Human Resource Management, 25(16), 2295-2318.

Ariss, A. A., \& Crowley-Henry, M. (2013). Self-initiated expatriation and migration in the management literature: Present theorizations and future research directions. Career Development International, 18(1), 78-96.

Bardin, L. (2011). Análise de conteúdo. São Paulo: Edições 70.

Baruch, Y., Dickmann, M., Altman, Y., \& Bournois, F. (2013). Exploring international work: types and dimensions of global careers. The International Journal of Human Resource Management, 24(12), 2369-2393.

Biemann, T., \& Andresen M. (2010). Self-initiated foreign expatriates versus assigned expatriates: two distinct types of international careers? Journal of Managerial Psychology, 25(4), 430-448.

Brewster, C., Sparrow, P., \& Vernon, G. (2010). International Human Resource Management (2nd ed.). London: Chartered Institute of Personnel and Development.

Buch, T., Schmidt, T. D., \& Niebuhr, A. (2009). Cross border commuting in the Danish German border region integration, institutions and cross border interaction. Journal of Borderlands Studies, 24(2), 38-54.

Bueno, J. M. (2010). Brasileiros e estrangeiros na construção de um cotidiano organizacional intercultural. 314 f. Tese (Doutorado em Administração de Empresas) - Escola de Administração de Empresas de São Paulo, Fundação Getúlio Vargas, São Paulo.

Bueno, J. M., \& Freitas, M. E. (2015). As Equipes Multiculturais em Subsidiárias Brasileiras de Multinacionais: um estudo de casos múltiplos. Organizações \& Sociedade, 22(72), 15-34.

Calderón, P. A. L., Guedes, A. L. M., \& Carvalho, R. W. (2016). Gestão internacional de recursos humanos: adaptabilidade intercultural na expatriação de brasileiros. Internext-Revista eletrônica de negócios internacionais da ESPM, 11(2), 6-20.

Caligiuri, P. M., \& Colakoglu, S. (2007). A strategic contingency approach to expatriate assignment management. Human Resource Management Journal, 17(4), 393-410.

Cao, L., Hirschi, A., \& Deller, J. (2013). The positive effects of a protean career attitude for self-initiated expatriates: Cultural adjustment as a mediator. Career Development International, 18(1), 56-77.

Cerdin, J.-L. (2004). Les carrières dans un contexte global. Management \& Avenir, (1), 155-175.

Cerdin, J.-L., \& Selmer, J. (2014). Who is a self-initiated expatriate? Towards conceptual clarity of a common notion. The International Journal of Human Resource Management, 25(9), 1281-1301.

Cerdin, J.-L., \& Sharma, K. (2014). Inpatriation as a key component of global talent management. In Ariss, A. A. 
(Org.). Global talent management. Gewerbestrasse: Springer International Publishing.

Chen, Y.-P., \& Shaffer, M. A. (2017). The influences of perceived organizational support and motivation on selfinitiated expatriates' organizational and community embeddedness. Journal of World Business, 52(2), 197-208.

Collings, D. G., Scullion, H., \& Morley, M. J. (2007). Changing patterns of global staffing in the multinational enterprise: challenges to the conventional expatriate assignment and emerging alternatives. Journal of World Business, 42(2), 198-213.

Dalto, G. N., \& Santos, A. C. B. D. (2015). Novas concepções de carreira e fidelidade corporativa: uma (im) possibilidade em tempos de capitalismo flexível? Revista de Carreiras e Pessoas, 5(3), 312-329.

Decoville, A., Durand, F., Sohn, C., \& Walther, O. (2013). Comparing Cross-border Metropolitan Integration in Europe: towards a functional typology. Journal of Borderlands Studies, 28(2), 221-237.

Dickmann, M., Doherty, N., Mills, T., \& Brewster, C. (2008). Why do they go? Individual and corporate perspectives on the factors influencing the decision to accept an international assignment. The International Journal of Human Resource Management, 19(4), 731-751.

Doherty, N., Richardson, J., \& Thorn, K. (2013). Self-initiated expatriation and self-initiated expatriates: clarification of the research stream. Career Development International, 18(1), 97-112.

Domingues, C. R. (2011). Políticas de carreiras para o gestor internacional: um estudo em empresas brasileiras internacionalizadas (Tese de Doutorado). Fundação Getúlio Vargas, São Paulo.

Freitas, M. E. (2005). Executivos brasileiros expatriados na França: uma contribuição aos estudos organizacionais interculturais. Relatório de pesquisa (Pós-Doutorado para concurso de professora titular da EAESP/FGV) Escola de Administração de Empresas de São Paulo, Fundação Getúlio Vargas, São Paulo.

Freitas, M. E. (2006). Expatriação de executivos. GV-executivo, 5(4), 48-52.

Freitas, M. E. (2009). A mobilidade como novo capital simbólico ou sejamos nômades. Revista Organizações \& Sociedade, 16(49), 247-264.

Gallon, S., \& Antunes, E. D. D. (2016). Processo de expatriação: um modelo com fases e práticas. Revista Eletrônica de Estratégia \& Negócios, 8(2), 54-85.

Gallon, S., \& Bitencourt, B. M. (2016). Programas trainee e expatriação como processos que evidenciam a importância estratégica da Gestão de Recursos Humanos. TPA - Teoria e Prática em Administração, 5(2), 140-166.

Gallon, S., Scheffer, A. B. B., \& Bitencourt, B. M. (2013). "Eu fui, voltei e ninguém viu”: um estudo sobre a expectativa de carreira após a repatriação em uma empresa brasileira. Cadernos EBAPE. BR, 11(1), 128-148..

Harvey, M., Kiessling, T., \& Moeller, M. (2011). Globalization and the inward flow of immigrants: Issues associated with the inpatriation of global managers. Human Resource Development Quarterly, 22(2), 177-194.

Harvey, M., Speier, C., \& Novicevic, M. M. (1999). The role of inpatriation in global staffing, The International Journal of Human Resource Management, 10(3), 459-476.

Hussain, T., \& Deery, S. (2018). Why do self-initiated expatriates quit their jobs: the role of job embeddedness and shocks in explaining turnover intentions. International Business Review, 27(1), 281-288.

Inkson, K., Arthur, M. B., Pringle, J., \& Barry, S. (1997). Expatriate assignment versus overseas experience: Contrasting models of international human resource development. Journal of World Business, 32(4), 351-368.

Maley, J., Moeller, M., \& Harvey, M. (2015). Strategic inpatriate acculturation: A stress perspective. International Journal of Intercultural Relations, 49, 308-321. 
Mayerhofer, H., Hartmann, L. C., \& Herbert, A. (2004). Career management issues for flexpatriate international staff. Thunderbird International Business Review, 46(6), 647-666.

Mckenna, S., \& Richardson, J. (2007). The increasing complexity of the internationally mobile professional. Cross Cultural Management: an international journal, 14(4), 307-320.

Muritiba, P. M. (2009). Estratégia internacional de gestão de pessoas nas multinacionais brasileiras (Tese Doutorado). Universidade de São Paulo, São Paulo.

Salleh, N. M., \& Koh, J. (2013). Analysing the functions of short-term expatriate assignments. Procedia-Social and Behavioral Sciences, 107, 34-42.

Suutari, V., \& Brewster, C. (2000). Making their own way: International experience through self-initiated foreign assignments. Journal of World Business, 35(4), 417-436.

Suutari, V., Brewster, C., Mäkelä, L., Dickmann, M., \& Tornikoski, C. (2018). The effect of international work experience on the career success of expatriates: a comparison of assigned and self-initiated expatriates. Human Resource Management, 57(1), 37-54.

Tahvanainen, M., Welch, D., \& Worm, V. (2005). Implications of short-term international assignments. European Management Journal, 23(6), 663-673.

Thorn, K. (2009). The relative importance of motives for international self-initiated mobility. Career Development International, 14(5), 441-464.

Welch, D. E., Welch, L. S., \& Worm, V. (2007). The international business traveller: a neglected but strategic human resource. The International Journal of Human Resource Management, 18(2), 173-183.

Wood JR, T., Tonelli, M. J., \& Cooke, B. (2011). Colonização e neocolonização da gestão de recursos humanos no Brasil (1950-2010). RAE-Revista de Administração de Empresas, 51(3), 232-243.

Zikic, J. (2015). Skilled migrants' career capital as a source of competitive advantage: implications for strategic HRM. The International Journal of Human Resource Management, 26(10), 1360-1381.

\section{Contato:}

Daniela Farah de Lima

E-mail: farah.daniela@yahoo.com.br

Carlos Roberto Domingues

E-mail: carlos.domingues@ufu.br

Submetido em: 14/01/2020

Revisado em: $23 / 09 / 2020$

Aprovado em: 07/12/2020 


\section{APÊNDICE I}

\section{Roteiro de Entrevista - Autores de referência e objetivos das perguntas}

\begin{tabular}{|c|c|c|}
\hline PERGUNTA DE ENTREVISTA & AUTORES & OBJETIVO \\
\hline $\begin{array}{l}\text { Quais são os tipos de mobilidade internacional } \\
\text { adotados pela organização? Há quanto tempo } \\
\text { é adotada / praticada a mobilidade? }\end{array}$ & $\begin{array}{l}\text { Cappelen; Janssens (2005) - Collings; } \\
\text { Scullion; Morley (2007) - Welch; Welch; } \\
\text { Worm (2007) }\end{array}$ & $\begin{array}{l}\text { Identificar as práticas das áreas de } \mathrm{RH} \text { das } \\
\text { subsidiárias no que diz respeito a mobilidade } \\
\text { internacional com contrato local. }\end{array}$ \\
\hline $\begin{array}{l}\text { Existe uma separação da área de Recursos } \\
\text { Humanos em termos de expatriados e } \\
\text { domésticos? Se sim, como está estruturada? }\end{array}$ & $\begin{array}{l}\text { Andresen; Biemann (2013) - Brewster; } \\
\text { Sparrow; Vernon (2010) - Bueno (2010) } \\
\text { - Caligiuri; Colakoglu (2007) - Cerdin; } \\
\text { Sharma (2014) - Domingues (2011) - } \\
\text { Homem; Tolfo (2008) - Muritiba (2009) }\end{array}$ & $\begin{array}{l}\text { Identificar as práticas das áreas de } \mathrm{RH} \text { das } \\
\text { subsidiárias no que diz respeito a mobilidade } \\
\text { internacional com contrato local. }\end{array}$ \\
\hline $\begin{array}{l}\text { No que diz respeito aos profissionais globais, } \\
\text { com a internacionalização da empresa, quais } \\
\text { os cargos são eletivos para a mobilidade } \\
\text { internacional com contrato local? Quais são os } \\
\text { principais objetivos a serem alcançados com a } \\
\text { mobilidade? }\end{array}$ & $\begin{array}{l}\text { Biemann; Andresen (2010) - Brewster; } \\
\text { Sparrow; Vernon (2010) }\end{array}$ & $\begin{array}{l}\text { Comparar as práticas de } \mathrm{RH} \text { das } \\
\text { subsidiárias que trabalham com a mobilidade } \\
\text { internacional com contratos locais. }\end{array}$ \\
\hline $\begin{array}{l}\text { Quais são os processos e os critérios para } \\
\text { que o(a) profissional(is) possa }(\mathrm{m}) \text { participar ou } \\
\text { se candidatar à mobilidade internacional com } \\
\text { contrato local? }\end{array}$ & $\begin{array}{l}\text { Adler (2002) - Domingues (2011) - Freitas } \\
\text { (2009) - Salleh; Koh (2013) }\end{array}$ & $\begin{array}{l}\text { Analisar os efeitos das estratégias da área de } \\
\text { RH global com a intensificação dos contratos } \\
\text { locais para a mobilidade internacional, nos } \\
\text { RH das subsidiárias. }\end{array}$ \\
\hline $\begin{array}{l}\text { Existe um controle do processo de mobilidade } \\
\text { internacional por parte do RH? Se sim, como } \\
\text { ele é feito? }\end{array}$ & Bueno; Freitas (2015) & $\begin{array}{l}\text { Comparar as práticas de RH das } \\
\text { subsidiárias que trabalham com a mobilidade } \\
\text { internacional com contratos locais. }\end{array}$ \\
\hline $\begin{array}{l}\text { Como se dá o gerenciamento dos processos } \\
\text { que envolvem os profissionais globais, por } \\
\text { parte da Gestão Internacional de RH? }\end{array}$ & Brewster; Sparrow; Vernon (2010) & $\begin{array}{l}\text { Analisar os efeitos das estratégias da área de } \\
\text { RH global com a intensificação dos contratos } \\
\text { locais para a mobilidade internacional, nos } \\
\text { RH das subsidiárias. }\end{array}$ \\
\hline $\begin{array}{l}\text { Como se dá a gestão da carreira? } \\
\text { E no caso do autoiniciado com contrato local? }\end{array}$ & $\begin{array}{l}\text { Adler (2002) - Brewster; Sparrow; Vernon } \\
\text { (2010) - Craide; Silva (2012) - Dickmann } \\
\text { et al. (2008) - Domingues (2011) - Freitas } \\
\text { (2009) - Gallon; Schefer; Bitencourt } \\
\text { (2013) - Stahl; Miller; Thung (2002) } \\
\end{array}$ & $\begin{array}{l}\text { Analisar os efeitos das estratégias da área de } \\
\text { RH global com a intensificação dos contratos } \\
\text { locais para a mobilidade internacional, nos } \\
\text { RH das subsidiárias. }\end{array}$ \\
\hline $\begin{array}{l}\text { Qual é o suporte que o RH oferece ao } \\
\text { autoiniciado? É diferente do suporte oferecido } \\
\text { ao expatriado "tradicional"? }\end{array}$ & $\begin{array}{l}\text { Ariss; Crowley-Henry (2013) - Andresen } \\
\text { et al. (2014) - Biemann; Andresen (2010) } \\
\text { - Brewster; Sparrow; Vernon (2010) - Cao; } \\
\text { Hirschi; Deller (2013) - Cerdin; Selmer } \\
\text { (2014) - Gallon; Antunes (2016) - Hussain; } \\
\text { Deery (2018) - Inkson et al. (1997) - } \\
\text { Suutari; Brewster (2000) }\end{array}$ & $\begin{array}{l}\text { Identificar as práticas das áreas de RH das } \\
\text { subsidiárias no que diz respeito à mobilidade } \\
\text { internacional com contrato local. }\end{array}$ \\
\hline $\begin{array}{l}\text { Quais são as principais dificuldades, ou } \\
\text { desafios, em relação ao processo de } \\
\text { mobilidade internacional dos tipos expatriação } \\
\text { autoiniciada com contrato local? Quais são os } \\
\text { encaminhamentos e as ações diante dessas } \\
\text { questões? }\end{array}$ & $\begin{array}{l}\text { Ariss; Crowley-Henry (2013) - Ariss; } \\
\text { Özbilgin (2010) }\end{array}$ & $\begin{array}{l}\text { Comparar as práticas de RH das } \\
\text { subsidiárias que trabalham com a mobilidade } \\
\text { internacional com contratos locais. }\end{array}$ \\
\hline $\begin{array}{l}\text { A partir de qual momento há o desvinculamento } \\
\text { da área de RH com este profissional? Há } \\
\text { um acompanhamento "póstransferência" na } \\
\text { mobilidade com contrato local? }\end{array}$ & Collings; Scullion; Morley (2007) & $\begin{array}{l}\text { Comparar as práticas de } \mathrm{RH} \text { das } \\
\text { subsidiárias que trabalham com a mobilidade } \\
\text { internacional com contratos locais. }\end{array}$ \\
\hline $\begin{array}{l}\text { Como você vê o futuro desse tipo de } \\
\text { mobilidade (expatriação autoiniciada com } \\
\text { contrato local) na organização? }\end{array}$ & $\begin{array}{l}\text { Araújo et al. (2012) - Bueno; Freitas } \\
\text { (2015) - Cao, Hirschi e Deller (2013) - } \\
\text { Cerdin e Selmer (2014) - Homem; Tolfo } \\
\text { (2008) - Salleh; Koh (2013) - Suutari; } \\
\text { Brewster (2000) - Suutari et al. (2018) }\end{array}$ & $\begin{array}{l}\text { Analisar os efeitos das estratégias da área de } \\
\mathrm{RH} \text { global com a intensificação dos contratos } \\
\text { locais para a mobilidade internacional, nos } \\
\mathrm{RH} \text { das subsidiárias. }\end{array}$ \\
\hline
\end{tabular}

